\title{
Mid-term evaluation of clinical and functional outcomes after arthroscopic medial longitudinal and bucket-handle meniscus repair
}

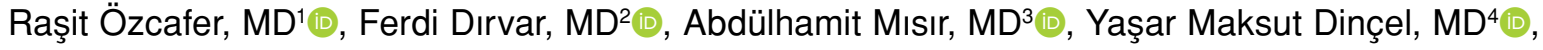 \\ Mehmet Özbey Büyükkuşçu, MD ${ }^{5}$, Ümit Selçuk Aykut, MD'이 \\ ${ }^{1}$ Department of Orthopedics and Traumatology, Vital Hospital, Istanbul, Turkey \\ ${ }^{2}$ Department of Orthopedics and Traumatology, Health Science University Metin Sabancı Baltalimani Bone Diseases Training and \\ Research Hospital, Istanbul,Turkey \\ ${ }^{3}$ Department of Orthopedics and Traumatology, Istanbul Başakşehir Çam ve Sakura City Training and Research Hospital, Istanbul, Turkey \\ ${ }^{4}$ Department of Orthopedics and Traumatology, Namık Kemal University, Faculty of Medicine, Tekirdağ, Turkey \\ ${ }^{5}$ Department of Orthopedics and Traumatology, Gaziosmanpaşa Training and Research Hospital, Istanbul, Turkey
}

The menisci have roles in shock absorption, proprioception, knee joint stability, and lubrication. ${ }^{[1]}$ Previously, it was demonstrated that contact pressure significantly increased even after partial meniscectomy. ${ }^{[2]}$ Meniscectomy has been associated with osteoarthritis in the long-term. ${ }^{[3]}$ Thus, repair of meniscal tears is recommended by most authors, particularly in young patients. ${ }^{[4]}$ Since partial meniscectomy predisposes to osteoarthritis, preserving menisci may reduce the risk of degeneration and disability. ${ }^{[5]}$

Longitudinal tears are common and are often repairable. ${ }^{[6]}$ Bucket-handle tears are complex and

Received: February 04, 2021

Accepted: April 21, 2021

Published online: June 11, 2021

Correspondence: Raşit Özcafer, MD. Vital Hastanesi Ortopedi ve Travmatoloji Bölümü, 34180 Bahçelievler, istanbul, Türkiye.

E-mail: rasitozcafer@yahoo.com

Doi: $10.52312 /$ jdrs.2021.46

Citation: Özcafer R, Dırvar F, Mısır A, Dinçel YM, Büyükkuşçu MÖ, Aykut ÜS. Mid-term evaluation of clinical and functional outcomes after arthroscopic medial longitudinal and buckethandle meniscus repair. Jt Dis Relat Surg 2021;32(2):363-370.

(02021 All right reserved by the Turkish Joint Diseases Foundation

This is an open access article under the terms of the Creative Commons Attribution-NonCommercial License, which permits use, distribution and reproduction in any medium, provided the original work is properly cited and is not used for commercial purposes (http://creativecommons.org/licenses/by-nc/4.0/).

\section{ABSTRACT}

Objectives: The aim of this study was to evaluate clinical and functional outcomes following the arthroscopic medial meniscal repair.

Patients and methods: A total of 50 patients (42 males, 8 females; mean age: $32.9 \pm 7.6$ years; range, 17 to 48 years) who underwent arthroscopic repair for longitudinal and bucket-handle medial meniscal tears between March 2005 and October 2011 were retrospectively evaluated. The patients were divided into two groups as those having a longitudinal tear (patient group, $n=31$ ) and having a bucket-handle tear (control group, $n=19$ ). Preoperative and final follow-up functional outcomes were evaluated using the Lysholm Knee Score (LKS), International Knee Documentation Committee (IKDC) score, Tegner Activity Scale (TAS) score, and Knee Injury and Osteoarthritis Outcome Score (KOOS).

Results: The mean follow-up was $61.7 \pm 22.8$ (range, 36 to 110 ) months. The mean preoperative LKS, IKDC score, TAS, and KOOS scores were significantly improved at the final postoperative follow-up $(\mathrm{p}<0.05)$. There was no significant difference in functional outcome scores between longitudinal and bucket-handle repairs ( $p>0.05)$, and isolated repairs and concomitant meniscal repair and anterior cruciate ligament reconstruction $(\mathrm{p}>0.05)$.

Conclusion: Arthroscopic meniscal repair provides similar mid-term functional and clinical outcomes for longitudinal and bucket-handle medial meniscal tears. Concomitant meniscal repair does not seem to affect meniscal healing.

Keywords: Anterior cruciate ligament, International Knee Documentation Committee, Lysholm Knee Score, meniscal repair, meniscal tear, meniscus. 
larger in size with displacement. Partial meniscectomy and meniscal repair are the treatment choices for both longitudinal and bucket-handle meniscal tears. ${ }^{[7]}$

Meniscal repair provides the improvement of knee function and, pain and mechanical symptom relief. The all-inside and inside-out techniques are commonly used in the meniscal repairs. In the literature, no significant difference has been shown in clinical outcomes and failure rates between the two techniques. ${ }^{[8]}$ However, there are still controversies regarding the effect of age, sex, concurrent anterior cruciate ligament (ACL) reconstruction, chronicity, involving medial or lateral meniscus on meniscal healing rates. ${ }^{[1,9,10]}$

In the literature, satisfactory outcomes have been reported following longitudinal and bucket-handle meniscal repairs. ${ }^{[1,11,12]}$ In the present study, we aimed to evaluate the functional and clinical outcomes of the patients undergoing arthroscopic repair of longitudinal and bucket-handle tears with a mean five-year follow-up, based on the hypothesis that comparable clinical and functional outcomes would be obtained after longitudinal and bucket-handle meniscal repair.

\section{PATIENTS AND METHODS}

This single-center, retrospective study was conducted at University of Health Sciences, Baltalimani Bone Diseases Education and Research Hospital, Department of Orthopedics and Traumatology between March 2005 and October 2011. A total of 91 patients underwent arthroscopic meniscal repair surgery during the study period. Inclusion criteria were longitudinal and bucket-handle meniscal tears located in red-red or red-white zones, tears larger than $1 \mathrm{~cm}$ length and isolated tears or concomitant meniscal and ACL rupture. Exclusion criteria were radial, horizontal, flap and degenerative tears, patients underwent other procedures not related to the meniscal repair in the same session and, the presence of another pathology such as osteoarthritis, avascular necrosis, osteochondritis dissecans or rheumatoid arthritis. After exclusions, 50 patients (42 males, 8 females; mean age: $32.9 \pm 7.6$ years; range, 17 to 48 years) who fulfilled the inclusion criteria were included. The patients were divided into two groups as those having a longitudinal tear (patient group, $\mathrm{n}=31$ ) and having a bucket-handle tear (control group, $\mathrm{n}=19$ ). A written informed consent was obtained from each patient. The study protocol was approved by the Metin Sabanc1 Baltalimani Bone Diseases Training and Research Hospital Ethics Committee (No: 14.04.2021/506). The study was conducted in accordance with the principles of the Declaration of Helsinki.

Characteristics of patients and features of the tears were noted. Preoperative radiographs and magnetic resonance imaging (MRI) scans were evaluated. Operative video records were reviewed to determine the type, the length, the localization and the vascular zone of the meniscal tears. The repair technique, the type of the suture material, the number of the sutures, and the orientation of the sutures in the meniscal body (vertical, horizontal or oblique) were recorded.

\section{Surgical technique}

Surgery was performed under general anesthesia. Standard anteromedial and anterolateral portals were used. The meniscal tear was assessed for the localization, stability, and reparability using an examination probe. Displaced bucket-handle type meniscal tears were reduced with a probe. If the patient was found to have a repairable tear, an arthroscopic shaver was used to prepare the tear edges before the repair. A rasp was, then, used to generate capillary bleeding.

The PDS 2/0 (Ethicon Inc., NJ, USA) suture material was used for all-inside-out repairs. For inside-out technique, suture needles were passed through both ends of the meniscal tear using cannulated guides and taken out of the skin with meticulous attention to be as close as possible (a maximum distance of $1 \mathrm{~cm}$ ). Additional incisions were not used to protect saphenous nerve medially and peroneal nerve branches laterally in any patients. ${ }^{[13]}$ Instead, stab incisions were used to tie the knots on the joint capsule. Stab incisions and portal incisions were closed with a nylon suture at the end of the surgery.

All-inside repairs were performed arthroscopically using the devices Fast-fix ${ }^{\circledR}$ (Smith \& Nephew, Inc., MA, USA), Sequent (ConMed, Linvatec, Largo, FL, USA) and Meniscal Cinch (Arthrex, Naples; FL, USA) following the manufacturers' technical guidelines.

In patients with concomitant ACL injury, single bundle ACL reconstruction with hamstring autografts was performed at the same session with the meniscal repairs.

\section{Clinical and radiological evaluation}

The clinical evaluation was performed by a blinded orthopedic surgeon. The range of motion (ROM) and knee stability, the presence of effusion, joint line tenderness was evaluated, and McMurray test was performed. Knee stability was evaluated 
with Lachman and pivot-shift tests. Neurological examination was performed for possible nerve irritation or neuroma that may be occurred due to meniscal repair. Preoperative and final follow-up Lysholm Knee Score (LKS), International Knee Documentation Committee (IKDC) score, Tegner Activity Scale (TAS) score, and Knee Injury and Osteoarthritis Outcome Score (KOOS) were used for functional evaluation between longitudinal and bucket-handle tears. ${ }^{[14-17]}$

\section{Statistical analysis}

Statistical analysis was performed using the IBM SPSS version 22.0 software (IBM Corp., Armonk, NY, USA). Descriptive data were expressed in mean \pm standard deviation (SD) or median (min-max) for continuous variables and in number and frequency for categorical variables. The distribution of variables was evaluated using the Kolmogorov-Smirnov test. The analysis of the quantitative data was performed with independent t-test and Mann-Whitney $U$ test. The chi-square test was used to analyze the quantitative values, whereas the Fisher test was applied when the chi-square test was not appropriate. Spearman correlation analysis was performed for correlation analysis. The Wilcoxon signed-ranked test was performed for repeated measurements. A $p$ value of $<0.05$ was considered statistically significant.

\section{RESULTS}

The mean follow-up period was $61.7 \pm 22.8$ (range, 36 to 110) months. Right knee was operated

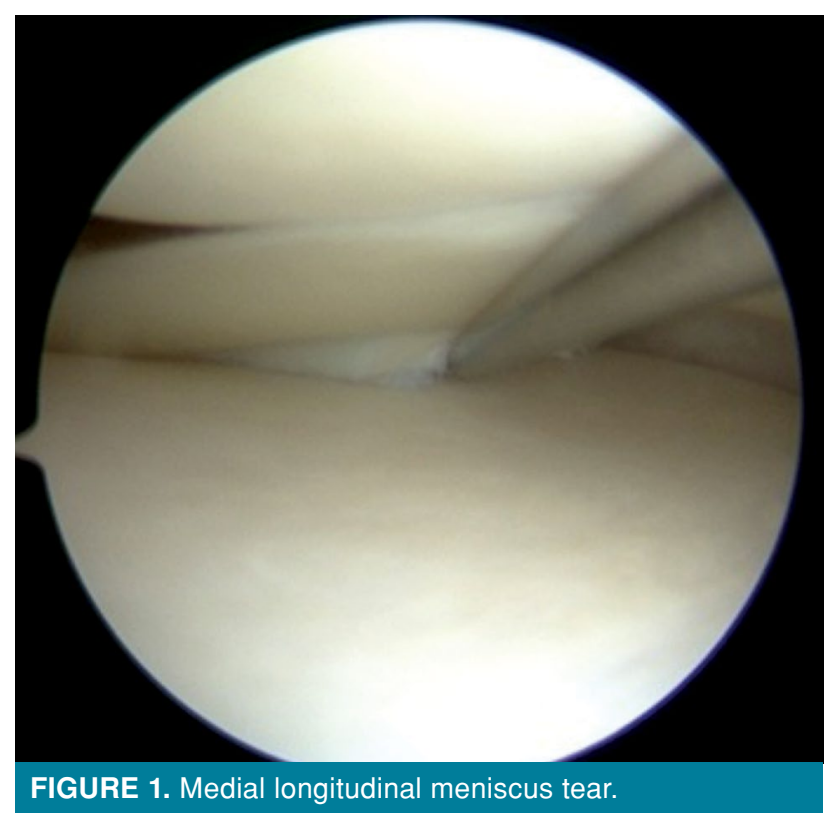

in $33(66 \%)$ patients, and left knee was operated in 17 (34\%). Medial meniscus was repaired in all patients. Concomitant ACL rupture was present in $30(60 \%)$ patients in which ACL reconstruction was performed in the same session with the meniscal repair. Twentyfour $(48 \%)$ patients were operated in the acute and subacute period (in the first 3 months after the initial trauma), whereas the remaining 26 (52\%) patients were operated in the chronic period (after the first three months). Forty-four (88\%) of the meniscal tears were localized in the red-red zone, while the remaining six
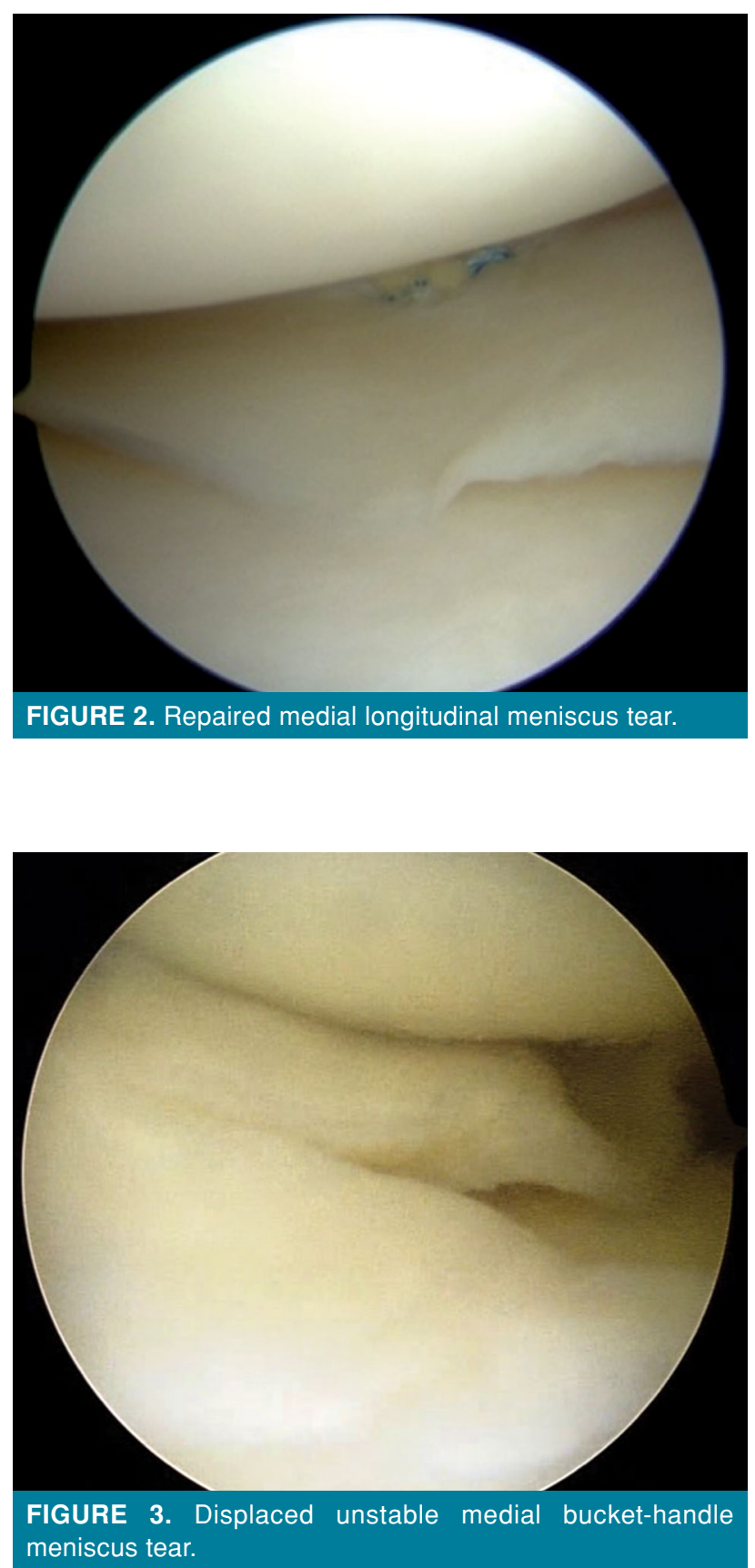

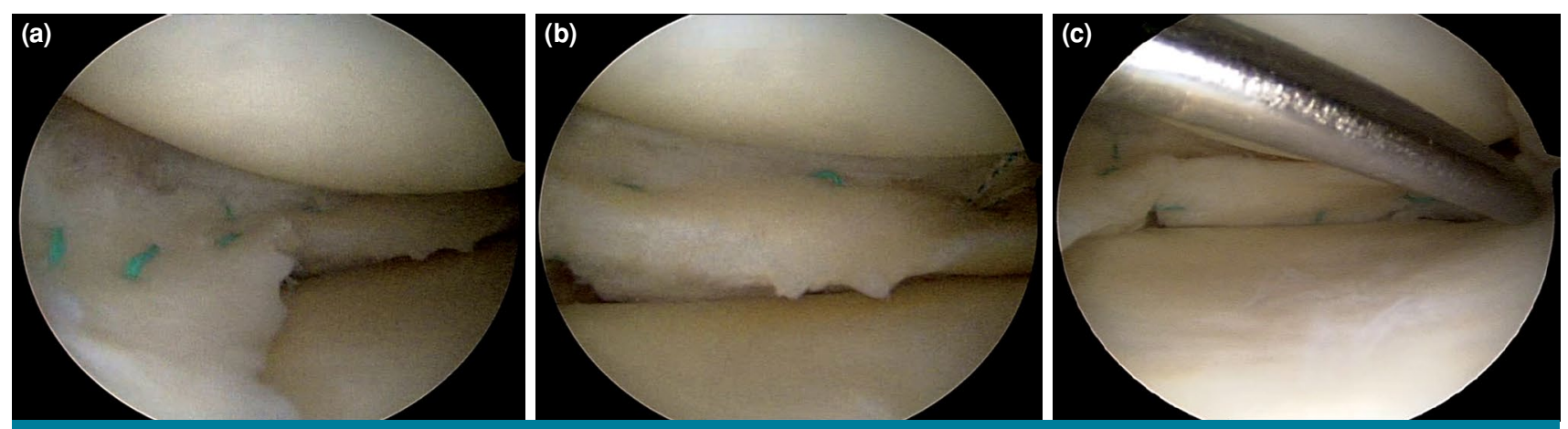

FIGURE 4. (a) Repaired medial bucket-handle meniscus tear from anterior horn to midbody. (b) Repaired medial bucket-handle meniscus tear from midbody to posterior. (c) Repaired medial bucket-handle meniscus tear of the posterior horn.

$(12 \%)$ tears were in the red-white zone. The meniscal tear type was longitudinal in $31(62 \%)$, and bucket handle type in 19 (38\%). Inside-out meniscal repair was performed in $46(92 \%)$ patients. All-inside repair was performed in four $(8 \%)$ patients, and inside-out repair combined with all-inside technique was performed in eight (16\%) patients (Figures 1-4). The mean number of the sutures performed was 3.6 \pm 2.4 (range, 2 to 11). The mean period between the onset of the complaints and surgery was $13.1 \pm 16.9$ (range, 0 to 120) months (Table I).

There was a statistically significant improvement in the LKS, IKDC, TAS, and KOOS scores postoperatively $(24.1 \pm 19.5$ vs. $85.0 \pm 12.2,23.8 \pm 10.1$ vs. $74.6 \pm 21.3,2.9 \pm 1.4$ vs. $4.9 \pm 1.9$ and $37.6 \pm 14.8$ vs. $79.9 \pm 14.6$, respectively) $(\mathrm{p}<0.05)$ (Table II). According to the results, LKS was perfect (80-100 points) in 38 patients
(62.29\%), good (70-79 points) in 17 patients $(27.86 \%)$, fair (60-69 points) in three patients $(4.91 \%)$, and poor ( $<60$ points) in three patients $(4.91 \%$ ). The outcomes of the latter three $(6 \%)$ patients considered as treatment failure.

There was no significant difference between bucket-handle and longitudinal tears regarding pre- and postoperative LKS, IKDC, TAS, and KOOS scores (Table III). Additionally, there was no significant difference between isolated repairs and concomitant meniscal repair and ACL reconstruction groups regarding functional outcomes $(\mathrm{p}>0.05)$.

Postoperative paresthesia in the medial side of the cruris was developed in eight patients. Of these, inside-out technique was performed in six patients,

\begin{tabular}{|c|c|c|c|c|c|c|c|c|c|c|}
\hline & & & Pat & $\begin{array}{l}\text { TAE } \\
\text { nt che }\end{array}$ & $\begin{array}{l}\text { E I } \\
\text { cterist }\end{array}$ & & & & & \\
\hline & Lor & tudina & roup $(n=31)$ & Bucl & t-hand & group $(n=19)$ & & & & \\
\hline & $\mathrm{n}$ & $\%$ & Mean \pm SD & $\mathrm{n}$ & $\%$ & Mean $\pm S D$ & $\mathrm{n}$ & $\%$ & Mean $\pm S D$ & $p$ \\
\hline Age (year) & & & $33.9 \pm 7.2$ & & & $30.7 \pm 7.4$ & & & $32.2 \pm 8.3$ & 0.235 \\
\hline Sex & & & & & & & & & & 1.000 \\
\hline Female & 5 & 16.1 & & 3 & 15.7 & & 8 & 16 & & \\
\hline Male & 26 & 83.9 & & 16 & 84.3 & & 42 & 84 & & \\
\hline ACL rupture & & & & & & & & & & \\
\hline Yes & 18 & 58 & & 12 & 63.1 & & 30 & 60 & & \\
\hline No & 13 & 42 & & 7 & 36.9 & & 20 & 40 & & \\
\hline Age of tear (month) & & & $28 \pm 26.8$ & & & $29.4 \pm 40$ & & & & 0.921 \\
\hline Zone of rupture & & & & & & & & & & 0.681 \\
\hline $\mathrm{RR}$ & 27 & 87.2 & & 19 & 100 & & 46 & 92 & & \\
\hline RW & 4 & 12.8 & & 0 & 0 & & 4 & 8 & & \\
\hline Length of tear (mm) & & & $3.1 \pm 0.7$ & & & $3.7 \pm 0.6$ & & & & 0.893 \\
\hline Suture number & & & $3.58 \pm 1.1$ & & & $4.3 \pm 3$ & & & $3.62 \pm 2.4$ & 0.821 \\
\hline
\end{tabular}




\begin{tabular}{|c|c|c|c|}
\hline & $\begin{array}{l}\text { TABLE II } \\
\text { - and postope }\end{array}$ & ve outcome sco & \\
\hline & Preoperative & Postoperative & \\
\hline & Mean $\pm S D$ & Mean $\pm S D$ & $p$ \\
\hline LKS & $24.1 \pm 19.5$ & $85.0 \pm 12.2$ & 0.001 \\
\hline TAS & $2.9 \pm 1.4$ & $4.9 \pm 1.9$ & 0.012 \\
\hline IKDC & $23.8 \pm 10.1$ & $74.6 \pm 21.3$ & 0.001 \\
\hline KOOS & $37.6 \pm 14.8$ & $79.9 \pm 14.6$ & 0.001 \\
\hline $\begin{array}{l}\text { SD: Sta } \\
\text { Internati } \\
\text { Score. }\end{array}$ & $\begin{array}{l}\text { Knee Score; } \\
\text { ttee; KOOS: Kn }\end{array}$ & $\begin{array}{l}\text { Tegner Activity } \\
\text { ry and Osteoarth }\end{array}$ & $\begin{array}{l}\text { e; IKDC } \\
\text { Outcome }\end{array}$ \\
\hline
\end{tabular}

\begin{tabular}{|c|c|c|c|}
\hline \multicolumn{4}{|c|}{$\begin{array}{l}\text { TABLE III } \\
\text { Pre- and postoperative outcome scores of longitudinal and bucket-handle tear groups }\end{array}$} \\
\hline & Longitudinal $(n=31)$ & Bucket-handle $(n=19$ & \\
\hline & Mean $\pm S D$ & Mean $\pm S D$ & $p$ \\
\hline Preoperative LKS & $23.0 \pm 21.4$ & $23.3 \pm 15.9$ & 0.470 \\
\hline Postoperative LKS & $62.0 \pm 21.2$ & $59.7 \pm 23.3$ & 0.529 \\
\hline Preoperative TAS & $3.0 \pm 1.7$ & $2.8 \pm 1.1$ & 0.975 \\
\hline Postoperative TAS & $5.1 \pm 1.8$ & $4.8 \pm 2.2$ & 0.559 \\
\hline Preoperative IKDC & $23.3 \pm 11.8$ & $24.0 \pm 8.2$ & 0.669 \\
\hline Postoperative IKDC & $72.4 \pm 25.4$ & $74.8 \pm 16.7$ & 0.797 \\
\hline Postoperative KOOS & $81.8 \pm 15.1$ & $78.8 \pm 14.8$ & 0.346 \\
\hline
\end{tabular}

and all-inside technique was performed in two patients.

\section{DISCUSSION}

The main finding of the present study was that repair of both longitudinal and bucket-handle meniscal tears caused improved clinical and functional outcomes with low failure rates. Outcomes of longitudinal and bucket handle tears were comparable regardless of concomitant ACL reconstruction or chronicity. According to our results, concomitant ACL reconstruction and chronic tear repair had no superiority over isolated repairs or acute tear repair. Our results are not consistent with various reports, ${ }^{[14,19,20]}$ but compatible with Uzun et al. ${ }^{[1]}$ and Nepple et al. ${ }^{[21]}$ Of note, in the literature, most studies evaluating the meniscal repair results have up to three-year follow-up results. ${ }^{[22]}$ Our study has a mean 61.7 months of follow-up.

Mid-term clinical and functional outcomes after arthroscopic meniscal repair for longitudinal and bucket-handle tears have been studied previously.
Similar functional outcome scores have been reported in most of the studies. Uzun et al. ${ }^{[23]}$ reported comparable functional outcomes for longitudinal and bucket-handle meniscal tear repair. They also found no significant difference for functional outcome between isolated repair and concomitant ACL reconstruction and meniscal repair. In their prospective observational study, Pande et al. ${ }^{[24]}$ evaluated functional outcome after arthroscopic longitudinal and bucket handle tear repairs in a military population. They found successful postoperative functional outcome scores with high return-to-duty rates. Our findings are consistent with these reports.

Buyukkuscu et al. ${ }^{[25]}$ reported $66.7 \%$ clinical improvement in patients older than 40 years and who underwent meniscal repair. Likewise, Noyes and Barber-Westin $^{[26]}$ reported $88 \%$ successful results in patients older than 40 years after repair of meniscal tears extending to the avascular region. We also believe that the meniscal repair should be performed in any age group and meniscal tissue should be 
maintained as much as possible with consideration of the physiological age of the patient, activity level, ligament stability and level of postoperative rehabilitation potential. In our study, we also obtained satisfactory functional results in patients over 40 years of age, as well as young patients, suggesting that meniscal repair can be performed in older ages.

Tuckman et al. ${ }^{[27]}$ found no significant difference in the healing of menisci between acute and chronic tears in at least two years of follow-up in series including 157 patients. Melton et al. ${ }^{[9]}$ also found no significant difference between acute and chronic meniscal repair outcomes in 44 patients. In our series, no statistically significant difference was found between the functional outcomes of meniscus repairs applied in the acute and subacute period and those applied in the chronic period. We believe that the tissue quality of injured meniscus is more important than the age of meniscal tears in this regard. We, therefore, suggest that the meniscus repair would be successful, if the location of the meniscal tear is in the vascular zone, reducible, and the torn meniscus has not been already degenerated.

In their systematic review including 13 studies with at least five-year follow-up, Nepple et al. ${ }^{[21]}$ found similar failure rates from the comparison of inside-out and all-inside repair techniques in meniscal repair. In our study, the inside-out technique was performed in 46 patients, while the all-inside technique was performed in four patients. In eight patients, a combined inside-out and all-inside technique was performed. There was no statistically significant difference among these groups regarding repair success, compatible with the current literature. The suture technique was preferred according to the pattern of the meniscal tear and the region in which it was located. We believe that all-inside technique is more useful, if one-third posterior of the meniscus is accessible, and all-inside or inside-out techniques are superior for repairing the middle-third portion of the meniscus and outside-in, or inside-out techniques are superior for repairing the anterior one-third portion of the meniscus.

Concomitant ACL reconstruction has been advocated in patients with coexistent ACL injury and meniscal due to the positive biomechanical contribution for meniscal healing. ${ }^{[28]}$ This surgery is associated with significant excessive intra-articular hemorrhage and fibrin clots within the joint space are thought to have a benefit on the healing process. ${ }^{[18]}$ DeHaven ${ }^{[29]}$ reported $100 \%$ success in 33 meniscus repair cases in ACL-intact knees after a 10.9-year follow-up, compared to $67 \%$ success in unstable knees. In our study, ACL reconstruction and meniscus repair were performed simultaneously in 30 patients, and isolated meniscal repair was performed in 20 patients. The increase in the postoperative IKDC scores was found to be higher in patients with meniscus repair with ACL reconstruction than the isolated meniscus repair. However, it did not reach statistical significance. Accordingly, knee stability and marrow stimulation may not be important in meniscal repair.

Complications of arthroscopic meniscus includes temporary saphenous nerve lesion, arthrofibrosis, temporary fibular nervelesion, infection, instrumental breakage, deep vein thrombosis, pulmonary embolism, meniscus cysts, reflex sympathetic dystrophy, and popliteal artery injury. Although posterior capsular dissection was used in the inside-out technique, nerve irritation was found to be 9\% in the literature. ${ }^{[30]}$ All-inside technique has lower nerve irritation rates from the inside-out, although $2 \%$ nerve irritation rates were found with all-inside technique. ${ }^{[30]}$ Westermann et al. ${ }^{[31]}$ also reported that the inside-out technique could cause significant local nerve damage, despite posterior capsular dissection. In our study, sensory hypoesthesia was detected in eight of 50 patients $(16 \%)$ in the innervation area of the saphenous nerve. Of these eight patients, inside-out repair technique was performed in six $(75 \%)$ and all-inside technique was performed in two $(25 \%)$. No painful neuroma was detected in any of these patients. Unlike the literature, posterior capsular dissection was not performed with the inside-out technique in our study. The suture ends were knotted on the capsule after reaching the capsule from the stab skin incisions. Nerve irritation was detected in six (13.04\%) out of 46 patients with inside-out technique. Although we did not use large posterior capsular dissection instead of a mini-incision, we believe that the rate of nerve complication is close to that in the literature.

The main limitations of our study are the use of heterogeneous repair techniques, and relatively small sample size including a heterogeneous patient population. Also, we were unable to evaluate meniscal healing with second look arthroscopy. Comparing the preoperative and the final follow-up outcomes also bears a bias for our study. Comparison of early postoperative and final follow-up scores would give more objective comparison. We obtained only postoperative KOOS scores. Lack of the preoperative KOOS brings additional bias for pre- and postoperative functional outcome change. 
In conclusion, meniscal repair provides good functional and clinical results at a mean five-year follow-up. Concomitant meniscal repair and ACL reconstruction may enhance meniscal healing. Nerve irritation is a complication that can be encountered during meniscus repair.

\section{Declaration of conflicting interests}

The authors declared no conflicts of interest with respect to the authorship and/or publication of this article.

\section{Funding}

The authors received no financial support for the research and/or authorship of this article.

\section{REFERENCES}

1. Uzun E, Misir A, Kizkapan TB, Ozcamdalli M, Akkurt S, Guney A. Arthroscopic medial meniscal repair with or without concurrent anterior cruciate ligament reconstruction: A subgroup analysis. Knee 2018;25:109-17.

2. Koh JL, Yi SJ, Ren Y, Zimmerman TA, Zhang LQ. Tibiofemoral contact mechanics with horizontal cleavage tear and resection of the medial meniscus in the human knee. J Bone Joint Surg [Am] 2016;98:1829-36.

3. Miao Y, Yu JK, Ao YF, Zheng ZZ, Gong X, Leung KK. Diagnostic values of 3 methods for evaluating meniscal healing status after meniscal repair: comparison among second-look arthroscopy, clinical assessment, and magnetic resonance imaging. Am J Sports Med 2011;39:735-42.

4. Noyes FR, Chen RC, Barber-Westin SD, Potter HG. Greater than 10-year results of red-white longitudinal meniscal repairs in patients 20 years of age or younger. Am J Sports Med 2011;39:1008-17.

5. Englund M, Roos EM, Lohmander LS. Impact of type of meniscal tear on radiographic and symptomatic knee osteoarthritis: A sixteen-year followup of meniscectomy with matched controls. Arthritis Rheum 2003;48:2178-87.

6. Moatshe G, Cinque ME, Godin JA, Vap AR, Chahla J, LaPrade RF. Comparable outcomes after bucket-handle meniscal repair and vertical meniscal repair can be achieved at a minimum 2 years' follow-up. Am J Sports Med 2017;45:3104-10.

7. Persson F, Turkiewicz A, Bergkvist D, Neuman P, Englund M. The risk of symptomatic knee osteoarthritis after arthroscopic meniscus repair vs partial meniscectomy vs the general population. Osteoarthr Cartil 2018;26:195-201.

8. Fillingham YA, Riboh JC, Erickson BJ, Bach BR Jr, Yanke $A B$. Inside-out versus all-inside repair of isolated meniscal tears: An updated systematic review. Am J Sports Med 2017;45:234-42.

9. Barber-Westin SD, Noyes FR. Clinical healing rates of meniscus repairs of tears in the central-third (red-white) zone. Arthroscopy 2014;30:134-46.

10. Melton JT, Murray JR, Karim A, Pandit H, Wandless F, Thomas NP. Meniscal repair in anterior cruciate ligament reconstruction: A long-term outcome study. Knee Surg Sports Traumatol Arthrosc 2011;19:1729-34.

11. Ahn JH, Kim KI, Wang JH, Kyung BS, Seo MC, Lee SH. Arthroscopic repair of bucket-handle tears of the lateral meniscus. Knee Surg Sports Traumatol Arthrosc 2015;23:205-10.
12. Espejo-Reina A, Serrano-Fernández JM, Martín-Castilla B, Estades-Rubio FJ, Briggs KK, Espejo-Baena A. Outcomes after repair of chronic bucket-handle tears of medial meniscus. Arthroscopy 2014;30:492-6.

13. Johnson D, Weiss B. Meniscal repair using the inside-out suture technique. Sports Med Arthrosc Rev 2012;20:68-76.

14. Tegner Y, Lysholm J, Lysholm M, Gillquist J. A performance test to monitor rehabilitation and evaluate anterior cruciate ligament injuries. Am J Sports Med 1986;14:156-9.

15. Irrgang JJ, Ho H, Harner CD, Fu FH. Use of the International Knee Documentation Committee guidelines to assess outcome following anterior cruciate ligament reconstruction. Knee Surg Sports Traumatol Arthrosc 1998;6:107-14.

16. Tegner Y, Lysholm J. Rating systems in the evaluation of knee ligament injuries. Clin Orthop Relat Res 1985;198:43-9.

17. Sivachidambaram K, Ateef M, Tahseen S. Correlation of Self-Reported Questionnaire (KOOS) with some objective measures in primary OA knee patients. ISRN Rheumatol 2014;2014:301485.

18. Atik OS. What are the expectations of an editor from a scientific article? Jt Dis Relat Surg 2020;31:597-8.

19. Wasserstein D, Dwyer T, Gandhi R, Austin PC, Mahomed N, Ogilvie-Harris D. A matched-cohort population study of reoperation after meniscal repair with and without concomitant anterior cruciate ligament reconstruction. Am J Sports Med 2013;41:349-55.

20. Toman CV, Dunn WR, Spindler KP, Amendola A, Andrish JT, Bergfeld JA, et al. Success of meniscal repair at anterior cruciate ligament reconstruction. Am J Sports Med 2009;37:1111-5.

21. Nepple JJ, Dunn WR, Wright RW. Meniscal repair outcomes at greater than five years: A systematic literature review and meta-analysis. J Bone Joint Surg [Am] 2012;94:2222-7.

22. Stärke C, Kopf S, Petersen W, Becker R. Meniscal repair. Arthroscopy 2009;25:1033-44.

23. Uzun E, Misir A, Kizkapan TB, Ozcamdalli M, Akkurt S, Guney A. Evaluation of midterm clinical and radiographic outcomes of arthroscopically repaired vertical longitudinal and bucket-handle lateral meniscal tears. Orthop J Sports Med 2019;7:2325967119843203.

24. Pande H, Padhi PP, Bhattacharya M. Functional outcome of arthroscopic repair of bucket handle and longitudinal medial meniscal tears in a military population by inside out and outside in technique: A prospective observational study. J Arthrosc Jt Surg 2020;7:137-44.

25. Buyukkuscu MO, Misir A, Hamrayev AJ, Ozcafer $\mathrm{R}$, Cetinkaya E. Clinical and radiological outcomes following isolated vertical medial meniscal tear repair in patients over 40 years old. J Orthop Surg (Hong Kong) 2019;27:2309499019836300.

26. Noyes FR, Barber-Westin SD. Arthroscopic repair of meniscal tears extending into the avascular zone in patients younger than twenty years of age. Am J Sports Med 2002;30:589-600.

27. Tuckman DV, Bravman JT, Lee SS, Rosen JE, Sherman OH. Outcomes of meniscal repair: minimum of 2-year followup. Bull Hosp Jt Dis 2006;63:100-4.

28. Kalliakmanis A, Zourntos S, Bousgas D, Nikolaou P. Comparison of arthroscopic meniscal repair results using 3 different meniscal repair devices in anterior cruciate ligament reconstruction patients. Arthroscopy 2008;24:810-6. 
29. DeHaven KE, Lohrer WA, Lovelock JE. Long-term results of open meniscal repair. Am J Sports Med 1995;23:524-30.

30. Grant JA, Wilde J, Miller BS, Bedi A. Comparison of insideout and all-inside techniques for the repair of isolated meniscal tears: A systematic review. Am J Sports Med 2012;40:459-68.
31. Westermann RW, Duchman KR, Amendola A, Glass $\mathrm{N}$, Wolf BR. All-inside versus inside-out meniscal repair with concurrent anterior cruciate ligament reconstruction: A meta-regression analysis. Am J Sports Med 2017;45:719-24. 